\title{
Exploring the Impact of Cognitive Style and Academic Discipline on Design Prototype Variability
}

\author{
Dr. Kathryn Jablokow, Pennsylvania State University
}

Dr. Kathryn Jablokow is an Associate Professor of Mechanical Engineering and Engineering Design at Penn State University. A graduate of Ohio State University (Ph.D., Electrical Engineering), Dr. Jablokow's teaching and research interests include problem solving, invention, and creativity in science and engineering, as well as robotics and computational dynamics. In addition to her membership in ASEE, she is a Senior Member of IEEE and a Fellow of ASME. Dr. Jablokow is the architect of a unique 4-course module focused on creativity and problem solving leadership and is currently developing a new methodology for cognition-based design. She is one of three instructors for Penn State's Massive Open Online Course (MOOC) on Creativity, Innovation, and Change, and she is the founding director of the Problem Solving Research Group, whose 50+ collaborating members include faculty and students from several universities, as well as industrial representatives, military leaders, and corporate consultants.

\section{Dr. Katja N Spreckelmeyer, Stanford University, Dept. of Psychology \\ Jacob Hershfield \\ Max Hershfield \\ Carolyn McEachern, Stanford University}

Carolyn McEachern is a third year undergraduate student at Stanford University, working towards a Bachelors of Science in Engineering: Product Design. Her focus is human-centered design, with an interest in user testing and prototyping.

\section{Prof. Martin Steinert Steinert, NTNU (Norwegian University of Science and Technology)}

Martin Steinert, Ph.D. is Professor of Eng. Design and Innovation at the Department of Eng. Design and Materials at the Norwegian University of Science and Technology (NTNU). I teach fuzzy front-end engineering for radical new product/service/system concepts and graduate research seminars for PhDs engaged in topics related to new product design and development. My various research projects are usually multidisciplinary (ME/CS/EE/Neuro- and Cognitive Sc.) and often connected with industry. The aim is to uncover, understand and leverage early stage engineering design paradigms with a special focus onto human-machine/object interactions. Recently I have published in Int. Journal of Product Development, Int. Journal of Design Creativity and Innovation, Journal of Eng. Design and Technology, Int. Journal of Design, Int. Journal of Eng. Education, Tech. Forecasting and Social Change, Energy Policy, Information Knowledge System Management Journal ... Ever since a short stint at MIT and my time as Deputy Director at the Center for Design Research and at the d.research program (Hasso Plattner Design Thinking Research program) at Stanford University, the overarching aim of my research and teaching is to always push the boundaries for Norwegian product development teams, so that they will ideate, more radical new concepts, faster.

\section{Prof. Larry Leifer, Stanford University, Center for Design Research}

Larry Leifer is a Professor of Mechanical Engineering Design and founding Director of the Center for Design Research (CDR) at Stanford University. He has been a member of the faculty since 1976. His teaching-laboratory is the graduate course ME310, "Industry Project Based Engineering Design, Innovation, and Development." Research themes include: 1) creating collaborative engineering design environments for distributed product innovation teams; 2) instrumenting that environment for design knowledge capture, indexing, reuse, and performance assessment; and 3), design-for-sustainable-wellbeing. His top R\&D priorities in the moment include the Hasso Plattner Design-Thinking-Research Program, d.swiss, and the notion of a pan-disciplinary $\mathrm{PhD}$ program in Design. 


\title{
Exploring the Impact of Cognitive Style and Academic Discipline on Design Prototype Variability
}

\begin{abstract}
This paper describes a pilot study in which we explored the impact of cognitive style and academic discipline on the variability of prototypes in design tasks as part of a larger research project aimed at understanding the relationships between design behavior, cognitive preferences, and physiological reactions. Engineering and non-engineering students were asked to complete a simple design, build, and test task using an egg-drop design challenge. The students' cognitive styles were assessed using the Kirton Adaption-Innovation Inventory (KAI); analysis revealed only slight differences between the engineering and non-engineering students in terms of cognitive style. Within-person comparisons of the similarity among built prototypes and the similarity between drawn and built prototypes were completed for each student; these results were correlated with discipline (engineering vs. non-engineering) and cognitive style to gain insight into their impact on students' design choices. Results of these analyses are discussed here, along with implications and limitations of this pilot study and our plans for future work in this domain.
\end{abstract}

\section{Research Context and Motivation}

This research is part of an NSF-funded collaborative project between Stanford University and Penn State University that spans the boundaries between engineering design and cognitive science $^{1}$ (see Figure 1). Our extended aim is to understand and model the relationships between engineering design behavior (actual engineering design activity), cognitive preferences (individual psychological predisposition), and real-time physiological responses (EEG, ECG, heart rate, etc.) during design. Our research focuses primarily on the early stages of product and engineering system design and development ${ }^{2,3}$, with potential for expansion across the entire design process.

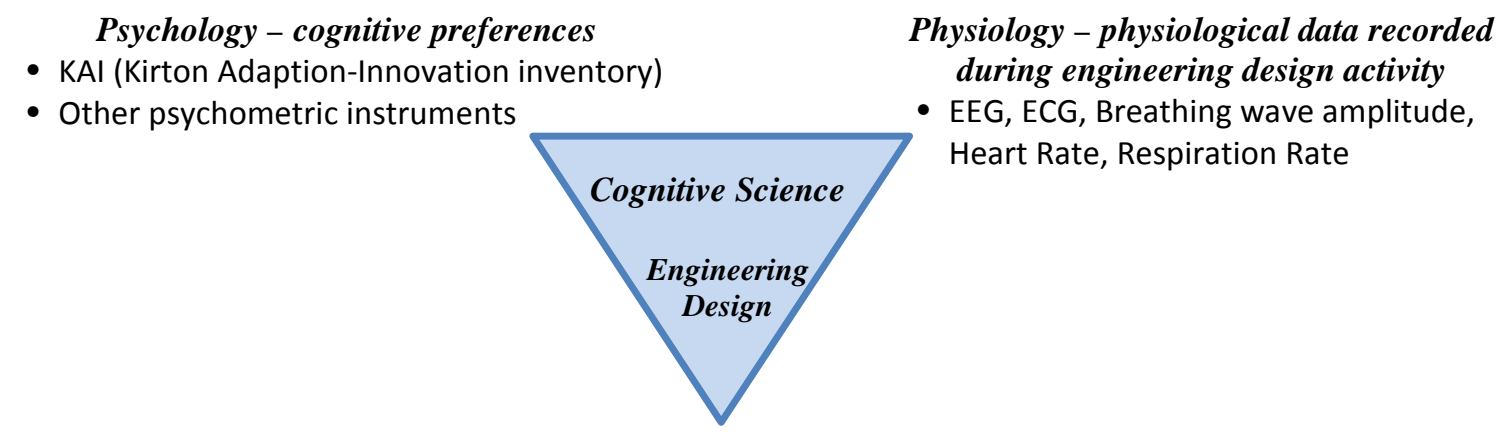

Behavior - engineering design activity

- In situ, controlled single subject engineering design challenge

- Video observation and recording

Figure 1. Key components of our research framework ${ }^{1}$

We have developed a testing scenario that involves multiple rounds of design activity for each subject, including ideation, paper-based and physical prototyping, testing, comparing, and 
ranking of design concepts. Each of these activities has clearly identifiable (and triggered) divergent and convergent phases. Our physiological measurements (e.g., EEG, ECG, heart rate, respiration) are focused on these divergent and convergent phases, from which we extract, analyze, and compare thin data slices of a few minutes each. In addition, we assess the cognitive preferences of each subject using the Kirton Adaption-Innovation Inventory (KAI) ${ }^{4,5}$, along with other validated psychometric instruments. Finally, we assess the design outcomes (concepts, drawings, prototypes) of each subject in various ways, depending on the particular aims of the research protocol ${ }^{6}$. All of these activities are video-recorded.

Through this range of experiments, we hope to gain insight into the impact of individual cognitive differences on the physiological reactions, design behaviors, and creativity of our students as they engage in design activities. Our long term aim is to use these insights to improve design education at our respective institutions by developing design instruction and prototyping techniques that can be individualized for each learner more effectively ${ }^{7,8,9,10}$.

\section{Previous Investigations and Current Research Questions}

The pilot study reported here is part of a series of pilot studies performed over the past twelve months at Stanford University. In mid-2013, we recruited small samples of engineering and nonengineering students and conducted several pilot studies using these samples to explore the research framework illustrated in Figure 1. As one example, we conducted an exploration of the physiological responses (e.g., EEG) of the subjects as they engaged in two simple design tasks (one divergent and one convergent) and correlated those results with their cognitive preferences (see Figure 2). The results of that pilot study revealed interesting differences in the EEG responses of individual subjects depending on whether they were engaged in a divergent or convergent task, but the sample sizes were too small to make any definite conclusions. We also observed varying amounts of individual stress associated with the divergent and convergent tasks depending on the cognitive styles of the subjects ${ }^{1}$.

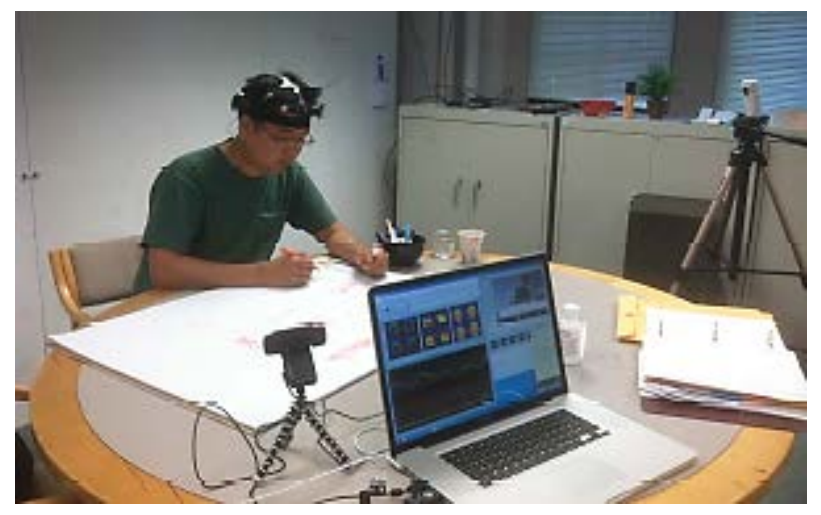

Figure 2. Previous pilot study investigating EEG responses and cognitive preferences of students engaged in simple design tasks

In the pilot study presented here, we explored relationships between the cognitive preferences of the subjects and their design behaviors - specifically, the variability of their designs within and between the various stages of the experiment. Reinertsen argues that variability is important for innovation and that a lack of variability can lead to less innovative outcomes ${ }^{11}$. In searching for ways to improve design education at our institutions, "design innovativeness" is a point of great 
interest to many faculty members. This fact led us to examine the variability of our subjects' design outcomes (i.e., concepts and prototypes) using the following research questions:

- Do engineers and non-engineers exhibit different amounts of variability in their designs?

- Do students of different cognitive styles exhibit different amounts of variability in their designs?

We note that other outcome metrics (e.g., quality, feasibility, novelty, etc.) might also be used to evaluate the design outcomes in this context, but since our aim here was focused more on the impact of individual differences on design decisions leading to (or away from) greater variability and less on the precise qualities of the design outcomes in an absolute sense, we chose to use a simpler evaluative metric for those outcomes.

\section{Experimental Methods}

\subsection{Study Participants}

Our sample included 23 student participants (mean age $=23.2$ yrs. \pm 4 yrs.); within this sample, 11 participants were female, 11 were male, and one student did not indicate gender. All were currently enrolled in different undergraduate programs at Stanford University. Participants were grouped into engineering students ( $\mathrm{N}=11$ : 3 female, 7 male, 1 unknown) and non-engineering students ( $\mathrm{N}=12: 8$ female, 4 male) based on their declared majors (see Table 1). Of the 23 participants, only 3 indicated that they had previous design experience (2 engineers and 1 nonengineer).

Table 1. Participants' declared majors (engineering vs. non-engineering)

\begin{tabular}{l|l|c}
\hline Engineering/Non-engineering & Declared Majors & Number of Participants \\
\hline \hline Engineering & Computer & 2 \\
& Electrical & 2 \\
& Industrial & 2 \\
& Mechanical & 3 \\
& Systems & 2 \\
\hline Non-engineering & Biology & 3 \\
& Business & 5 \\
& Economics & 2 \\
& Linguistics & 1 \\
& Physics & 1 \\
\hline
\end{tabular}

\subsection{Study Protocol}

Participants completed the Kirton Adaption-Innovation Inventory (KAI) ${ }^{4,5}$ before participating in the design task. In the task, participants were asked to first conceptualize, draw, and then build 3 different prototypes for a device that would allow them to drop a raw egg from different heights without the egg breaking (i.e., the classic egg-drop design challenge ${ }^{12}$ ). This particular design challenge was chosen based on its established procedure and its suitability for both engineering and non-engineering students alike; it was framed as a lander vehicle problem for added authenticity ${ }^{1}$. For prototyping, participants were provided with 3 identical sets of materials; each 
set included 1 plastic bag, 8 rubber bands, 8 pipe cleaners, 8 Popsicle sticks, a 4”x8” piece of foam core, a 4"x12” flat foam sheet, and 12” of tape. Participants had 10 minutes to conceptualize and draw their proposed prototypes (conceptualization phase) and 15 minutes to construct their prototypes (building phase). The testing phase did not have a time limit. In the sub-sections below, we will discuss each phase of the study protocol in more detail.

\subsubsection{Measuring cognitive style}

As mentioned above, in addition to noting differences in discipline (as shown in Table 1), we also assessed the cognitive style of each participant using the KAI (Kirton Adaption-Innovation Inventory), which is a highly validated psychometric instrument ${ }^{4,5}$. As measured by KAI, cognitive style lies on a bipolar continuum that ranges from strong Adaption on one end to strong Innovation on the other. In general, an individual's cognitive style is related to the amount of structure he or she prefers when solving problems (making decisions, processing information), with more adaptive individuals preferring more structure (with more of it consensually agreed), and more innovative individuals preferring less structure (with less concern about consensus). These differences lead to distinctive patterns of behavior, although an individual can and does behave in ways that are not preferred; this is called coping behavior ${ }^{5,13}$.

In addition to these broad differences, an individual's cognitive style can also be analyzed in terms of three sub-factors: Sufficiency of Originality (SO), Efficiency (E), and Rule/Group Conformity $(\mathrm{R} / \mathrm{G})^{5}$. Sufficiency of Originality (SO) highlights differences between individuals in their preferred ways of generating ideas. For example, the more adaptive prefer to offer a "sufficient" number of novel options in ideation that are readily seen to be relevant, acceptable, and aimed at immediate and efficient improvements to the current structure (system, solution, process, etc.). In contrast, the more innovative prefer to proliferate novel options in ideation, some (even many) of which may not be seen as immediately relevant to the current problem and/or may be difficult to implement as part of the current structure.

The Efficiency (E) sub-factor reflects an individual's preferred methods or tactics for managing ideas and solving problems. For example, the more adaptive prefer to define problems and their solutions carefully, paying closer attention to details while searching methodically for relevant information. They also tend to be more organized and meticulous in their operations. In contrast, the more innovative often loosen and/or reframe the definition of a problem before they begin to resolve it, paying less attention to detail and taking a seemingly casual and less careful approach as they search for and carry out their solutions.

The Rule/Group Conformity (R/G) sub-factor reflects differences in the ways individuals manage the personal and impersonal structures in which their problem solving occurs. For example, the more adaptive generally see standards, rules, traditions, instructions, and guidelines (all examples of "impersonal" structures) as enabling and useful, while the more innovative are more likely to see them as limiting and irritating. When it comes to personal structures (e.g., teams, partnerships), the more adaptive tend to devote more attention to group cohesion, while the more innovative are more likely to “stir up” a group’s internal dynamics. 
For large general populations and across cultures ${ }^{4,5}$, the distribution of KAI total scores forms a normal curve within the theoretical range of (32-160), with an observed mean of 95 and an observed range of (43-149); sub-scores corresponding to the three sub-factors are also normally distributed within the following theoretical ranges: SO (13-65), E (7-35), and R/G (12-60) ${ }^{4,5}$. The just noticeable difference (JND) for KAI between individuals is 10 points; that is, differences of only 10 points in total scores are noticeable over time, with larger style gaps (especially those greater than 20 points) being readily detected and potentially problematic. The just-noticeable-difference between group means for KAI is 5 points ${ }^{5,13}$. Jablokow's study of graduate engineering students showed wide ranges of KAI scores among systems engineers, software engineers, and information scientists ${ }^{7}$, while DeFranco et al. ${ }^{14}$ reported similar findings among undergraduate engineering students.

\subsubsection{Introduction to the problem}

To introduce the egg-drop design task, participants watched a real-life video showing the successful touchdown of a lander vehicle on the moon. In the video, it was pointed out that special technologies were required to achieve a soft, safe, and precise landing; in other words, the lander vehicle problem was used to give the egg-drop design task some real-world flavor. Participants were then instructed to conceptualize and build their own prototypes of lander vehicles that would allow the soft, safe landing of a breakable object (here, a raw egg) on a hard surface.

\subsubsection{Conceptualization phase}

Participants were presented with the building materials and asked to brainstorm possible uses for each individual item (e.g., the plastic bag, Popsicle sticks, pipe cleaners). They were given 10 minutes to write down or draw as many different uses as they could for each item. They were then asked to conceptualize at least 3 different full prototypes made of the given materials. Again, they could write down their ideas or draw them. This second conceptual step had to be completed in 5 minutes.

\subsubsection{Building phase}

Participants were provided with as many eggs and bags of materials as they needed to build their prototypes. However, only one bag of materials could be used for each prototype. Participants were given 15 minutes to complete their prototypes; the number of prototypes was not confined to 3 , but no participant completed more than 3 prototypes in the allotted time.

\subsubsection{Testing phase}

In the testing phase, the participants were asked to drop each of their prototypes from a height of 36" onto a wooden platform that recorded peak impact force, average impact force, and duration of impact. These force/impact data were not analyzed as part of this study; a successful test was determined simply according to whether the egg survived the drop without cracking or breaking. 


\section{Outcome Assessment and Analysis}

\subsection{Productivity and Performance Measures}

The number of built prototypes and the number of prototypes that survived the egg-drop testing procedure served as outcome measures to assess participants' productivity and performance, respectively. In addition, a variability/similarity rating technique was used to monitor decisionmaking during the design process and to gain two different measures of creative output, as described below.

\subsection{Prototype Assessment}

The development and application of outcome-based design metrics has been studied extensively by Shah, et al. ${ }^{15,16}$, Vargas-Hernandez, et al. ${ }^{17}$, Nelson, et al. ${ }^{18}$, and Verhaegen, et al. ${ }^{19}$, (among others), who have focused primarily on the use of these metrics to assess the effectiveness of design ideation methods. These metrics could certainly be applied in our context as well, but for the purposes of this pilot study, in which establishing the impact of individual differences on design decisions was the primary goal, the systematic but somewhat involved metrics of Nelson, Shah, and others were deemed overly complex. Instead, a simpler (albeit less comprehensive) procedure was used to evaluate variability among prototypes, as described below.

\subsubsection{Similarity between Built Prototypes}

For each participant, a pairwise comparison was performed of all his/her completed prototypes to assess conceptual variability/similarity among built prototypes. Comparisons were conducted by three independent raters (all engineering research assistants with at least one year of design experience) using a 5-point scale (1=low similarity to 5 =high similarity); see Figure 3 below for examples of low and high similarity. Inter-rater-reliability was 0.5 (Cohen's kappa); for the final analysis, the ratings were averaged across participants. The number of raters was determined using standard best practices in inter-rater reliability ${ }^{20}$.

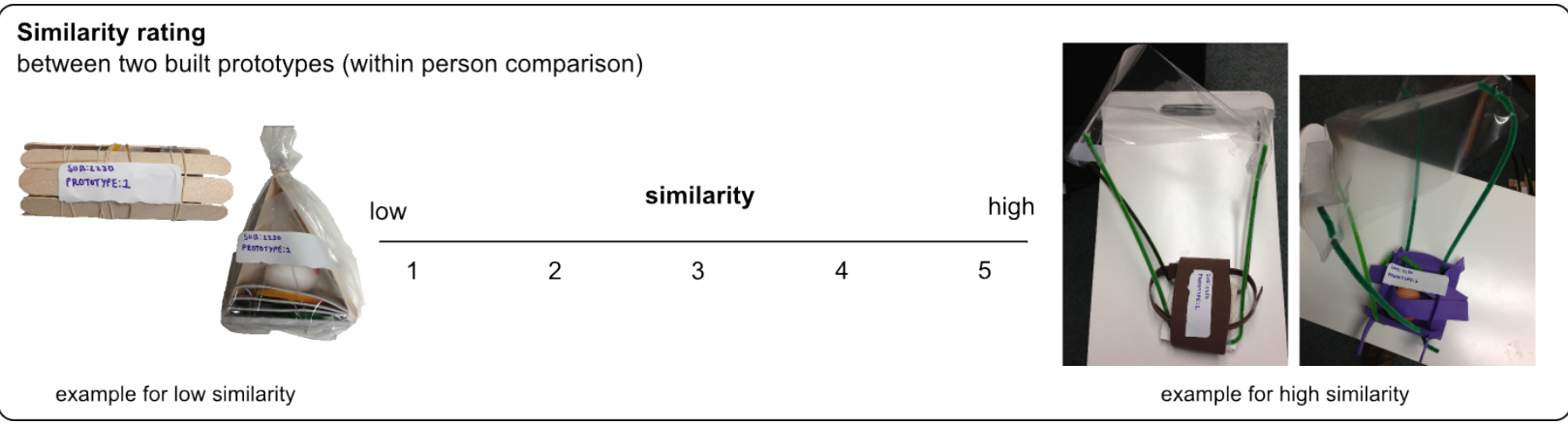

Figure 3. Illustration of similarity rating between built prototypes (within person comparison)

\subsubsection{Deviation of Built Prototypes from Idea Sketches}

In addition to similarity between the completed prototypes of each participant, we also assessed how much participants' built prototypes deviated from their original ideas as sketched/drafted in the conceptualization step of the experiment. In other words, we wanted to identify features that 
appeared in the built prototype that were not present in the conceptual prototype. Variability of ideas was defined as any addition that occurred in the built prototype that had not previously been drawn or described in writing. The same 5-point comparison scale was used as above (1=low similarity to 5=high similarity); see Figure 4 below for illustration. Again, comparisons were conducted by three independent raters (engineering research assistants with at least one year of design experience), with a resulting Cohen's kappa $=0.4$. The ratings were averaged across participants.

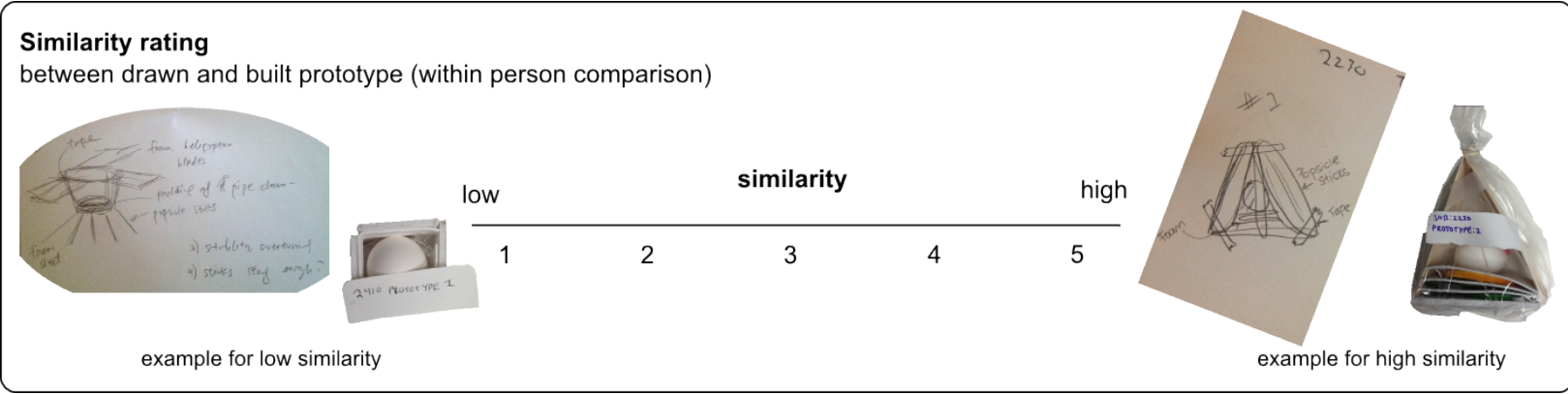

Figure 4. Illustration of similarity rating between drawn and built prototypes (within person comparison)

\section{Experimental Results}

\subsection{Individual Differences}

In general, our analysis revealed no statistically significant differences between non-engineering and engineering students with regard to cognitive style, although the KAI score distributions for the two samples were not identical (see Figures 5 and 6). As shown, means of the KAI total scores were 97.5 for engineers vs. 97.8 for non-engineers, respectively, a difference which is well within the 5-point just-noticeable-difference for KAI between groups ${ }^{5}$. The total score ranges were slightly different between the two groups (37 points vs. 58 points, respectively).

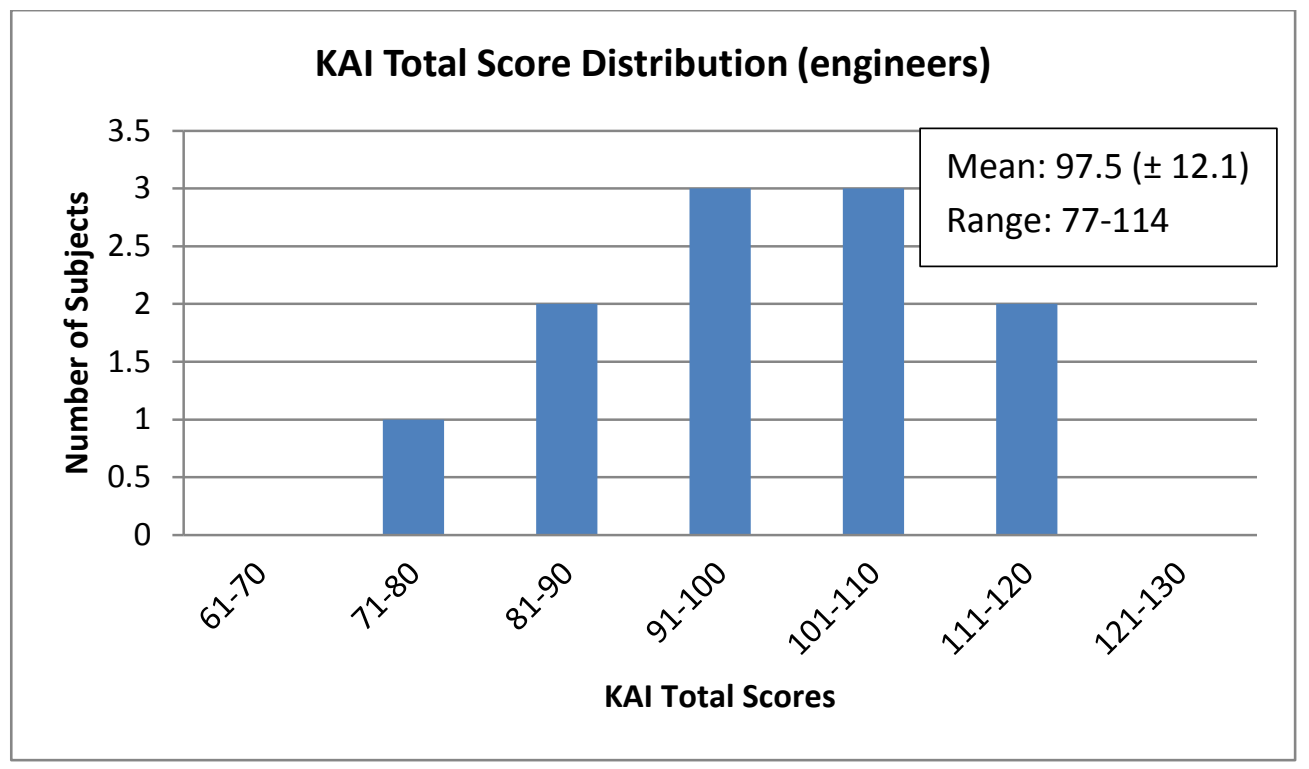

Figure 5. KAI total score distribution for engineering subjects $(\mathrm{N}=11)$ 


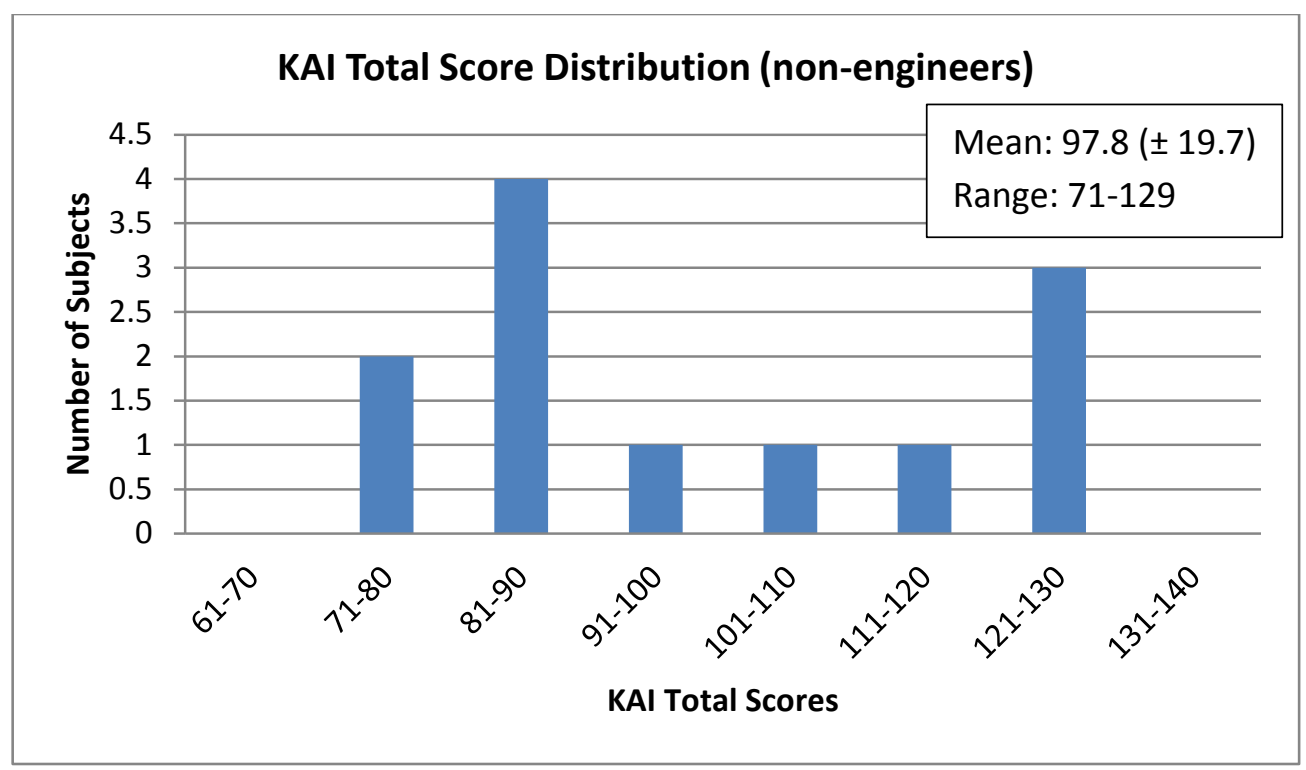

Figure 6. KAI total score distribution for non-engineering subjects $(\mathrm{N}=12)$

\subsection{Productivity and Performance}

Generally, the engineering and non-engineering groups were equally productive in terms of built prototypes, with averages of 2.2 (engineers) vs. 2.1 (non-engineers) completed prototypes per person, respectively. Individuals in the two groups also did not differ significantly with regard to performance, although non-engineering students tended to be more successful than engineering students overall (61\% vs. $41 \%$ of completed prototypes prevented the egg from breaking). When correlating cognitive style with prototype productivity and performance, some interesting results emerged. Across the two groups (all subjects), the number of completed prototypes correlated negatively with participants’ KAI total scores $(r=-0.478, p=0.021)$, as shown in Table 2 .

Table 2. Correlations between cognitive style, productivity, and performance (bold type indicates a statistically significant correlation)

\begin{tabular}{l|cc|cc|cc|cc}
\hline & \multicolumn{2}{|c|}{ KAI total score } & \multicolumn{2}{c|}{ SO sub-score } & \multicolumn{2}{c|}{ E sub-score } & \multicolumn{2}{c}{ R/G sub-score } \\
& $p$ value & Corr. $r$ & $p$ value & Corr. $r$ & $p$ value & Corr. $r$ & $p$ value & Corr. $r$ \\
\hline \hline All Subjects: & & & & & & & & \\
Completed prototypes & 0.021 & $\mathbf{- 0 . 4 7 8}$ & 0.281 & -0.235 & 0.001 & $\mathbf{- 0 . 6 3 7}$ & 0.028 & $\mathbf{- 0 . 4 5 9}$ \\
Number of broken eggs & 0.183 & -0.288 & 0.854 & -0.041 & 0.016 & $\mathbf{- 0 . 4 9 7}$ & 0.15 & -0.31 \\
\hline Engineers: & & & & & & & & \\
Completed prototypes & 0.044 & $\mathbf{- 0 . 6 1 6}$ & 0.947 & -0.023 & 0.001 & $\mathbf{- 0 . 8 4 6}$ & 0.039 & $\mathbf{- 0 . 6 2 6}$ \\
Number of broken eggs & 0.873 & 0.055 & 0.074 & 0.559 & 0.096 & -0.527 & 0.778 & -0.097 \\
\hline Non-engineers: & & & & & & & & \\
Completed prototypes & 0.167 & -0.426 & 0.172 & -0.421 & 0.203 & -0.396 & 0.219 & -0.383 \\
Number of broken eggs & 0.12 & -0.474 & 0.141 & -0.451 & 0.086 & -0.515 & 0.185 & -0.41 \\
\hline
\end{tabular}


These results suggest that the more adaptive participants were more likely to achieve the goal of 3 prototypes than the more innovative participants. This is also illustrated in Figure 7. The correlations between KAI total score, E sub-score, R/G sub-score, and completed prototypes were even stronger for the engineers alone (see Table 2), while no significant correlations were observed for the non-engineers alone. In the end, our samples are too small to make any conclusions, but our results may indicate a useful direction for future investigation.

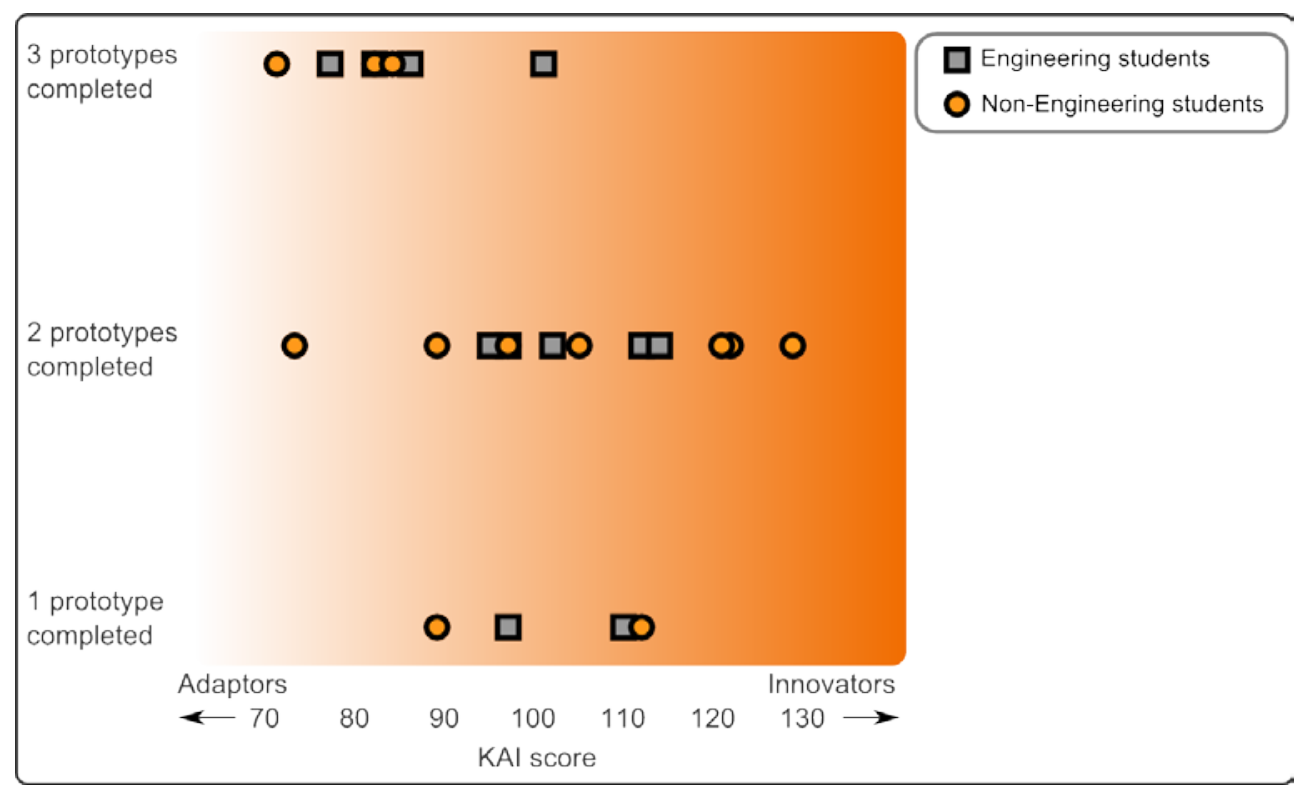

Figure 7. Number of completed prototypes vs. KAI total score (cognitive style)

Although these sample sizes are small, we can hypothesize about reasons that might lead to the results we observed. In general, individuals who are more adaptive tend to be more sensitive and more responsive to the "rules" of a problem or task - i.e., to the conditions that define success in the current context ${ }^{5,9}$. In this case, participants were specifically asked to produce at least 3 prototypes, which our more adaptive participants would be more likely to see as a hard requirement than a soft guideline (as their more innovative counterparts are likely to do). To confirm or refute this reasoning, we will need to interview or survey participants after the fact, to determine how strongly they felt driven by this task condition in their prototyping behavior and decision making. We plan to pursue this line of investigation in future work, as it may be relevant in determining how best to frame design problems for our students.

\subsection{Similarity between Built Prototypes}

Overall, our measures of variability/similarity among built prototypes revealed less variation than expected in both the engineering and non-engineering groups. Similarity between first and second built prototypes was rated intermediate across each group: 2.7 for non-engineers vs. 2.9 for engineers, respectively (see Figure 8 for individual ratings). No correlations were found between cognitive style (KAI total score, sub-scores) and similarity between built prototypes. 


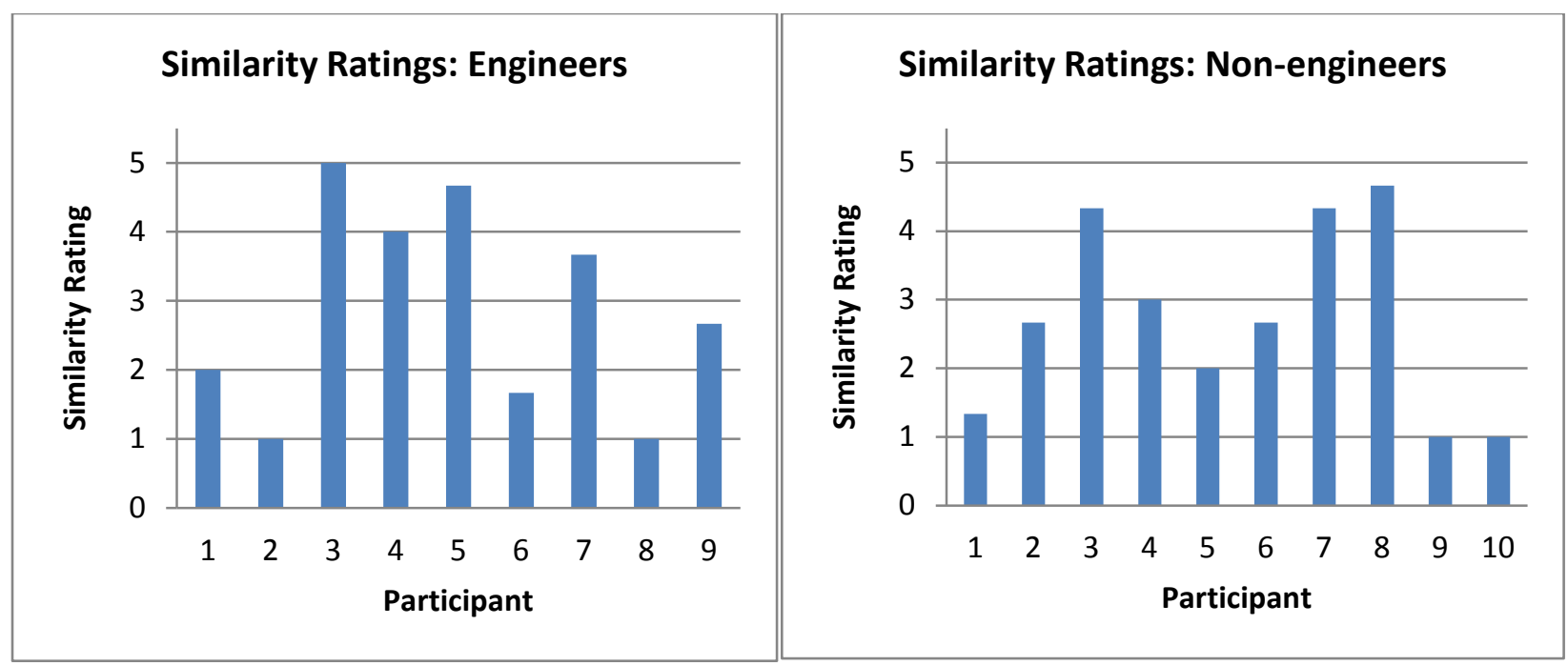

Figure 8. Similarity ratings (between built prototypes) for engineers and non-engineers

\subsection{Similarity between Built Prototypes and Idea Sketches}

Deviation of participants' built prototypes from their original idea sketches was found to be low in both non-engineering and engineering students (3.9 for engineers vs. 4.1 for non-engineers). Even so, some potentially interesting results emerged when we examined the correlations between cognitive style (KAI) and such variations (see Table 3). Among the engineering students, there were no strong correlations between cognitive style and the similarity between drawn and built design prototypes, although the relation between similarity and the SO sub-score was significant at the $90 \%$ level. For the non-engineers, however, positive correlations were found between all facets of cognitive style (the KAI total score and all three sub-scores) and these similarity ratings. In other words, the more innovative non-engineers were more likely to build prototypes that were similar to their drawn design concepts. Again, our sample sizes are too small to make any firm conclusions about these results, but we plan to pursue this line of investigation in future research.

Table 3. Correlations between cognitive style and drawn-built prototype similarity (bold type indicates significant correlation)

\begin{tabular}{|c|c|c|c|c|c|c|c|c|}
\hline & \multicolumn{2}{|c|}{ KAI total score } & \multicolumn{2}{|c|}{ SO sub-score } & \multicolumn{2}{|c|}{ E sub-score } & \multicolumn{2}{|c|}{ R/G sub-score } \\
\hline & $p$ value & Corr. $r$ & $p$ value & Corr. $r$ & $p$ value & Corr. $r$ & $p$ value & Corr. $r$ \\
\hline Engineers: & & & & & & & & \\
\hline Similarity: Drawn vs. built prototypes & 0.244 & -0.384 & 0.098 & -0.524 & 0.694 & 0.134 & 0.305 & -0.341 \\
\hline Non-engineers: & & & & & & & & \\
\hline Similarity: Drawn vs. built prototypes & 0.006 & 0.743 & 0.021 & 0.655 & 0.016 & 0.675 & 0.006 & 0.742 \\
\hline
\end{tabular}




\section{Conclusions, Implications, and Future Work}

As a reiteration of the main findings of this pilot study, we note the following:

- No significant statistical differences were found between the non-engineering and engineering students with respect to cognitive style.

- The engineering and non-engineering groups were equally productive in terms of built prototypes.

- The two groups did not differ significantly with regard to performance, although the nonengineering students tended to be more successful than the engineering students.

- Across the two groups, the number of completed prototypes correlated negatively with participants’ KAI total scores (i.e., with more adaptive cognitive style).

- Similarity between first and second built prototypes was rated as intermediate across both groups.

- Deviation of participants' built prototypes from their original idea sketches was found to be low in both non-engineering and engineering groups.

While the small sample sizes used in this study and the potential subjectivity of the three raters may limit our conclusions, the results are encouraging and suggest further investigation. Additional data collection is underway with expanded samples of both engineering and nonengineering students at both institutions. In summary, the results of this investigation revealed less variability between prototypes than we expected for both engineers and non-engineers. This suggests that more practice in prototyping (i.e., "making things") is still needed in the classroom, perhaps through prototyping challenges similar to the one featured here.

\section{Acknowledgements}

This work was supported by the National Science Foundation (NSF), Civil, Mechanical and Manufacturing Innovation (CMMI) Division, EAGER Grant \#1153823: AnalyzeD - Analyzing Engineering Design Activities. Any opinions, findings, conclusions, or recommendations expressed here are those of the authors and do not necessarily reflect the views of the National Science Foundation.

\section{References}

1. Steinert, M. and Jablokow, K. 2013. Triangulating front end engineering design activities with physiology data and psychological preferences. Proc. of 2013 International Conference on Engineering Design (ICED13), Seoul, Korea.

2. Dym, C. L., and Little, P. 2004. Engineering Design-A Project-based Introduction. MIT Press.

3. Cross, N. 2000. Engineering design methods: strategies for product design (Vol. 58). Wiley: Chichester.

4. Kirton, M. J. 1999. Kirton Adaption-Innovation Inventory Manual ( ${ }^{\text {rd }}$ ed.), Occupational Research Centre, Newmarket, Suffolk, U.K.

5. Kirton, M. J. 2011. Adaption-Innovation in the Context of Diversity and Change, London: Routledge. 
6. Skogstad, P., Steinert, M., Gumerlock, K., and Leifer, L.J., 2009. We need a universal design project outcome performance measurement metric: A discussion based on empirical research. Proc. of the 17th International Conference on Engineering Design (ICED’09), 6: 473-484.

7. Jablokow, K. W. 2008. Developing problem solving leadership: A cognitive approach. International Journal of Engineering Education, 25(5): 936-954.

8. Leifer, L. J., and Steinert, M. 2011. Dancing with ambiguity: Causality behavior, design thinking, and tripleloop-learning. Information, Knowledge, Systems Management, 10(1), 151-173.

9. Samuel, P., and Jablokow, K. W. 2011. Toward an Adaption-Innovation Strategy for Engineering Design. Proc. of the 18th International Conference on Engineering Design (ICED11), 2: 377-386.

10. Dym, C.L., Agogino, A.M., Eris, O., Frey, D.D., and Leifer, L.J. 2005. Engineering design thinking, teaching, and learning. Journal of Engineering Education, 95(1): 103-120.

11. Reinertsen, D. G. 2009. The Principles of Product Development Flow. Redondo Beach, CA: Celeritas Publishing.

12. Dow, S. P., Heddleston, K. and S. R. Klemmer. 2009. The efficacy of prototyping under time constraints. Source, Proc. C\&C-09, Berkeley, CA, 165-174, ACM 978-1-60558-403-4/09/10.

13. Jablokow, K. W. and M. J. Kirton. 2009. Problem solving, creativity, and the level-style distinction. Perspectives on the Nature of Intellectual Styles (L.-F. Zhang and R. J. Sternberg, Eds.), New York: Springer, 137-168.

14. DeFranco, J.F., Jablokow, K.W., Bilen, S.G., and A. Gordon. 2012. The Impact of Cognitive Style on Concept Mapping: Visualizing Variations in the Structure of Ideas. Proc. of the ASEE 2012 Annual Conference \& Exposition, San Antonio, TX.

15. Shah, J.J., Kulkarni, S.V. and N. Vargas-Hernandez. 2000. Evaluation of idea generation methods for conceptual design: effectiveness metrics and design of experiments. Journal of Mechanical Design, 122: 377384.

16. Shah, J. J., Smith, S. M., and N. Vargas-Hernandez. 2003. Metrics for measuring ideation effectiveness. Design Studies, 24(2): 111-134.

17. Vargas-Hernandez, N., Shah, J. J., and S. M. Smith. 2010. Understanding design ideation mechanisms through multilevel aligned empirical studies. Design Studies, 31(4): 382-410.

18. Nelson B. A., Wilson J. O., Rosen D., and Yen J. 2009. Refined metrics for measuring ideation effectiveness. Design Studies, 30(6): 737-743.

19. Verhaegen P.-A., Vandevenne D., Peeters J., and Duflou J. R. 2013. Refinements to the variety metric for idea evaluation. Design Studies, 34(2): 243-263.

20. Gwet, K. L. 2012. Handbook of Inter-Rater Reliability. Advanced Analytics, LLC; 3rd edition. 